El par les moindres quarres

$$
\begin{array}{r}
12 D-1,12 \frac{e^{2}}{2} D-23169,46=0 \\
-1,12 D+0,15 \frac{e^{2}}{2} D+2161,68=0
\end{array}
$$

d'où $\frac{\bullet^{2}}{2} D=+1 \gamma^{\prime \prime} 33 ; D=1932^{\prime \prime} 42 ;$ l'aplatissement du

Soleil aux poles = Ito; et les différences entre les diamètres horizontaux, observés ct culculées respectivement, résultent

$$
\begin{aligned}
\text { obserr_calc. } & =-0,30=+0,28=-2,66=+0,39 \\
& =+0,45=+3,58=-2,61=-0,84 \\
& =+0,78=+0,85=-1,62=+1,75 \\
\text { Moyenne } \ldots . .=+0,004 &
\end{aligned}
$$

Essayons encore une autro voie; tt un dénolant par $H$ le diamètre horizontal, par $V$ le verical, prenons pour $I S$ ct pour $\mathscr{V}$ les milieux respectifs de nos observations, et l'on aura $H=1930^{\prime \prime} 788 ; \quad F=1924^{\prime \prime} 515$. Or étant à peu près

$$
H=D-\frac{e^{2}}{2} D \frac{\Sigma p_{2}^{2}}{12} ; \quad \gamma=D-\frac{e^{2}}{2} D \frac{\Sigma p^{2}}{12},
$$

puiaque nous ayons $\frac{\Sigma p_{2}^{2}}{12}=0,093$, et $\frac{\Sigma p^{2}}{12}=0,898$ il en résultera par les substitutions et l'élimination

$\frac{e^{3}}{2}-D=+7$ 739; $D=1931^{\prime \prime} 465 ;$ etl'aplatissesnent $=$ कोषे Par l'ensemble donc de mes observations on obtient la G:ure du Suleil aplatie aux poles; el si lon veut on pourra en fixer poiur la qnantité plus procho av vrni aplatissement la moyenne des détcrminutions où nous sommes parvenus, qui est $=\frac{1}{2}$. J'ai dit par l'ensemble des observations; car si dans cette recherche du rapport des diamètres on y emploie les observations particllement de quelcpues mois, il peut arriver qu'on en trouve des résullats tout à fait upposés c'est-à-dire par quelques observations la figure aplatie et par des autres obserwations la figure allongée. Pour exemple si on applique la méthode que nous versons Je suivre aux diamètres borizontaux observél des mois de Janvier, Arril, Juillet et Oclobre, il en résultera l'aplatissement $=16$, qui s'accorde bien avec le présuédents valeurs: au contraire $i$ on traile pareillement et pum les qualre mémes unois les diamètres verticaux olosprvds, il en viendra un allongenent aux poles $=$ rt8. Tout cela signifie, il me semble, que la recherche est bicn delicate et qu'il faut hien en saisir les élémens el les conditions de probabilitú les plns favorables. Il convient pàr ce molif là de s'en tenir an plus grand nombre d'olservations ef ainsi inilopterois l'aplatissement क्षेत d'autant plus quil est peu diffírent du résullat obtenu par Mr. Carlini avec de mésures micromèlviques, lesquelles daus ce genre de déterminations sont préférablce à toul autre moyeu. Au reste je ne suis entré dans cel argument que pour vous en offrij un pelit essai, et pour exciter l'oltenlion el la criniosilé de quelqu'un à le poursuivre.

\section{J. Bianchi.}

Auszug aus einem Schreiben des Herrn Dwerhagen, an Herrn Dr. und Ritier Olbers in Bremen. Buenos Ayres. 1831. März 29.

(Von Ilerrn Dr. Ollers güligst raitgetheilt.)

Icis Labe die Ebre ihnen meine Beohachtungen des Cometen an iibersenden, welcher zuerst am siidlichen Hinmel 1830 den 18 keu Mlärz erschien, und bei denen mir meiz Frouud Herr Kiernau behülflicls war.

Wir glanbten erst dafs er den Astronomen anf dem Vorgehinges der guten Hoflunug, der unstreitig mit bessern Instrumenten ansgeriistet ist, nicht entgehen werle; da wir aber nock richts you dort gehört haben. so schien "nns doch die Mittheilung dessen, was wir, freilich nit geringeren Milteln, erhallen bahen, nicht ohne lnteresse zu seyn.
Ea komunt dazu dafs unsere Brobachtungen in den eaglischen Zeitungen so fehlerbalt unil extstellt beknnt gemacht sind, dafs ich, schon damit Sie richt qine zin schlechte Mejnung vou unserer Arbcit haben möditeu, Ihnen die Originale sende.

Die ersten Beobachtungen sind, glanibe ich, dir hesten, weil bald las Licht des Connter ledentead almahm. Lr hatle übrigens keinen Ecbwir, und bestand hlos aus einem Kerne, der sirh ron eebr schwackem Haar umgeben zevigte. 